\title{
The ginsenoside PPD exerts anti- endometriosis effects by suppressing estrogen receptor-mediated inhibition of endometrial stromal cell autophagy and NK cell cytotoxicity
}

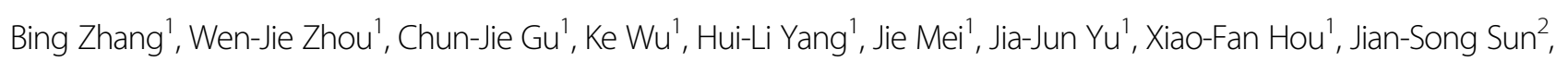
Feng-Yuan $\mathrm{Xu}^{3}$, Da-Jin Li ${ }^{1}$, Li-Ping $\mathrm{Jin}^{4}$ and Ming-Qing Li ${ }^{1,5}$

\begin{abstract}
Endometriosis (EMS) is an estrogen-dependent gynecological disease with a low autophagy level of ectopic endometrial stromal cells (eESCs). Impaired NK cell cytotoxic activity is involved in the clearance obstruction of the ectopic endometrial tissue in the abdominopelvic cavity. Protopanaxadiol (PPD) and protopanaxatriol (PPT) are two metabolites of ginsenosides, which have profound biological functions, such as anti-cancer activities. However, the role and mechanism of ginsenosides and metabolites in endometriosis are completely unknown. Here, we found that the compounds PPD, PPT, ginsenoside-Rg3 (G-Rg3), ginsenoside-Rh2 (G-Rh2), and esculentoside A (EsA) led to significant decreases in the viability of eESCs, particularly PPD $(I C 50=30.64 \mu \mathrm{M})$. In vitro and in vivo experiments showed that PPD promoted the expression of progesterone receptor (PR) and downregulated the expression of estrogen receptor a (ERa) in EESCs. Treatment with PPD obviously induced the autophagy of eESCs and reversed the inhibitory effect of estrogen on eESC autophagy. In addition, eESCs pretreated with PPD enhanced the cytotoxic activity of NK cells in response to eESCS. PPD decreased the numbers and suppressed the growth of ectopic lesions in a mouse EMS model. These results suggest that PPD plays a role in anti-EMS activation, possibly by restricting estrogen-mediated autophagy regulation and enhancing the cytotoxicity of NK cells. This result provides a scientific basis for potential therapeutic strategies to treat EMS by PPD or further structural modification.
\end{abstract}

\section{Introduction}

Endometriosis (EMS) is a common estrogen-dependent gynecological disease that is defined by the attachment of

\footnotetext{
Correspondence: M-Q. Li (mqli@fudan.edu.cn) or L-P. Jin (jinlp01@163.com) ${ }^{1}$ Laboratory for Reproductive Immunology, Key Laboratory of Reproduction Regulation of NPFPC, SIPPR, IRD, Hospital of Obstetrics and Gynecology, Fudan University Shanghai Medical College, 200011 Shanghai, China

${ }^{2}$ National Research Centre for Carbohydrate Synthesis, Jiangxi Normal University, 330022 Jiangxi, Nanchang, China

Full list of author information is available at the end of the article. These authors contributed equally: Bing Zhang and Wen-Jie Zhou Edited by B. Zhivotovsky
}

endometrial tissue at extrauterine ectopic sites where it forms invasive lesions ${ }^{1-3}$. It affects $\sim 5-15 \%$ of all women of reproductive age and $20-50 \%$ of all infertile women ${ }^{4,5}$. There is strong evidence to support the important role of estrogen dependence, progesterone resistance, and defects in the immune system (e.g., impaired cytotoxic activity of NK cells) in EMS, leading to the enhanced benign tumor-like behaviors of refluxed endometrium (such as unrestrained proliferation and aggressive invasion $)^{6-9}$.

\section{(c) The Author(s) 2018}

(c) Open Access This article is licensed under a Creative Commons Attribution 4.0 International License, which permits use, sharing, adaptation, distribution and reproduction c. in any medium or format, as long as you give appropriate credit to the original author(s) and the source, provide a link to the Creative Commons license, and indicate if changes were made. The images or other third party material in this article are included in the article's Creative Commons license, unless indicated otherwise in a credit line to the material. If material is not included in the article's Creative Commons license and your intended use is not permitted by statutory regulation or exceeds the permitted use, you will need to obtain permission directly from the copyright holder. To view a copy of this license, visit http://creativecommons.org/licenses/by/4.0/. 
Current medical therapies (e.g., progestins, androgens, gonadotropin-releasing hormone $(\mathrm{GnRH})$ agonists, and aromatase inhibitors) focus primarily on reducing the systemic levels of estrogens, but these are of limited effectiveness, with frequent recurrence and considerable side effects ${ }^{1}$. Therefore, the most urgent need is to better understand the mechanisms underlying EMS, to enable the development of more effective treatments.

As a constitutive catabolic pathway, autophagy is the natural, regulated, destructive mechanism of the cell that mediates both non-specific and targeted sequestration of cellular organelles and other macromolecules, allowing the orderly degradation of cellular components in lysosomes and the recycling of bioenergetics metabolites ${ }^{10}$. Accumulating evidence has indicated that the level of autophagy in both the ectopic and eutopic endometrium of patients with EMS is decreased ${ }^{7,11-15}$. This aberrantly low autophagy is associated with the highly proliferative and lowly apoptotic capacities of ectopic endometrial stromal cells $(\mathrm{eESCs})^{7,11-14}$. Our previous work has showed that estrogen promotes the survival of human ESCs via upregulating CXCL12/CXCR4-mediated autophagy inhibition ${ }^{7}$. The CXCR4-CXCL12 axis has also been shown to have both immune (e.g., lymphocyte chemotaxis, especially for NK cells) and non-immune functions (e.g., tissue repair, angiogenesis, cell invasion, and migration $)^{16,17}$. In addition, our recent study has observed that the low autophagy of ESCs leads to the impaired cytotoxicity of NK cells in a co-culture system, which may be beneficial to the immune escape of ectopic ESCs ${ }^{8}$. Therefore, a new therapeutic strategy should be urgently required, that is, targeting autophagy and immunomodulation simultaneously.

As a traditional medicinal herb, ginseng is widely used in Asian countries and North America. Ginsenosides (e.g., ginsenoside- Rg3 (G-Rg3) and ginsenoside Rh2 (G-Rh2)) are the main components extracted from ginseng and have various pharmaceutical activities, such as antitumor, antioxidant, immunomodulatory, and anti-inflammatory activities $^{18-21}$. As two metabolites of ginsenoside, protopanaxadiol (PPD) and protopanaxatriol (PPT) also exhibit activity against various cancer cells $\mathrm{s}^{20,22,23}$. Various functional assays and molecular docking studies have provided evidence that ginsenosides can mediate their cellular activities by binding to the active sites of steroid receptors (e.g., estrogen receptor $\alpha(E R \alpha))^{20}$. However, to date, no study has been published regarding the anti-EMS (a benign estrogen-dependent disease with tumor-like behaviors) activities of these ginsenosides and metabolites.

Therefore, the aim of this study was to investigate whether ginsenosides and metabolites have anti-EMS activity, and if so, whether this effect is dependent on the regulation of autophagy and ER in eESCs and the cytotoxicity of NK cells in vitro and in vivo.

\section{Results}

\section{PPD has powerful anti-EMS activity in vitro}

Esculentoside A (EsA), a triterpene saponin isolated from the root of the Chinese herb Phytolacca esculenta, is known for its anti-inflammatory and anti-oxidative effects $^{24}$. It has been reported that EsA inhibits the growth of ectopic lesions in a rat EMS model, and its effect is stronger than that of the same dose of azole (a synthetic steroid that is used primarily in the treatment of EMS) (China Patent Number: CN200510110906.X). The chemical structures of PPD, PPT, G-Rg3, G-Rh2 and EsA are shown in Figure 1a. Owing to the anti-EMS activity and similar chemical structures among EsA, ginsenosides and metabolites, EsA was used as a positive control. First, we evaluated the effects of PPD, PPT, G-Rg3, G-Rh2, and EsA on eESCs and normal ESCs (nESCs) in vitro. As shown, treatment with PPD, PPT, G-Rh2, or EsA led to a significant decrease in eESC viability, especially at concentrations beyond $40 \mu \mathrm{M}$ (Fig. 1b). Among these compounds, PPD has the most powerful inhibition on eESC viability, with an $\mathrm{IC}_{50}$ (half maximal inhibitory concentration) of $30.64 \mu \mathrm{M}$ (Fig. 1c). However, none of these compounds (PPD, PPT, G-Rh2, or EsA) showed a significant effect on the viability of nESCs unless their concentrations were higher than $80 \mu \mathrm{M}$ (Supplementary Figure 1). Further analysis showed that the expression levels of anti-apoptosis molecules B-cell lymphoma (Bcl)$\mathrm{xL}$ and $\mathrm{Bcl}-2$, and proliferation-related molecules Ki-67 and proliferating cell nuclear antigen (PCNA) in PPDtreated eESCs were obviously decreased compared with those of control eESC and EsA-treated eESCs (Fig. 1d, e). Conversely, the tumor metastasis suppressor CD82 ${ }^{25}$ and pro-apoptotic molecules Bax and Bak were increased (Fig. 1d-f). These results indicated that a low level of PPD can markedly inhibits the viability of eESCs not nESCs in vitro.

\section{PPD suppresses estrogen-mediated autophagy inhibition of eESCs possibly by downregulating ERa and upregulating progesterone receptor $a(P R a)$ in vitro}

To investigate the potential effect of PPD on the autophagy of eESCs, quantitative real time polymerase chain reaction (qRT-PCR)-based analysis of 84 genes involved in autophagy (a human autophagy PCR array) was performed (Fig. 2a). As shown, 21 different genes (such as autophagy-related genes (ATG)2, ATG3, and ATG5, ESR1, SQSTEM1, and TGFB1) between control eESCs and PPD-treated eESCs (the differential fold $>3$ in eESCs) was found (Fig. 2b). The results of western blotting showed that PPD upregulated autophagy-related molecules microtubule-associated protein light chain 3B (LC3B)-II, Beclin-1, and downregulated p62 expression in eESCs (Fig. 2c), suggesting that PPD could enhance the autophagy of eESCs. 


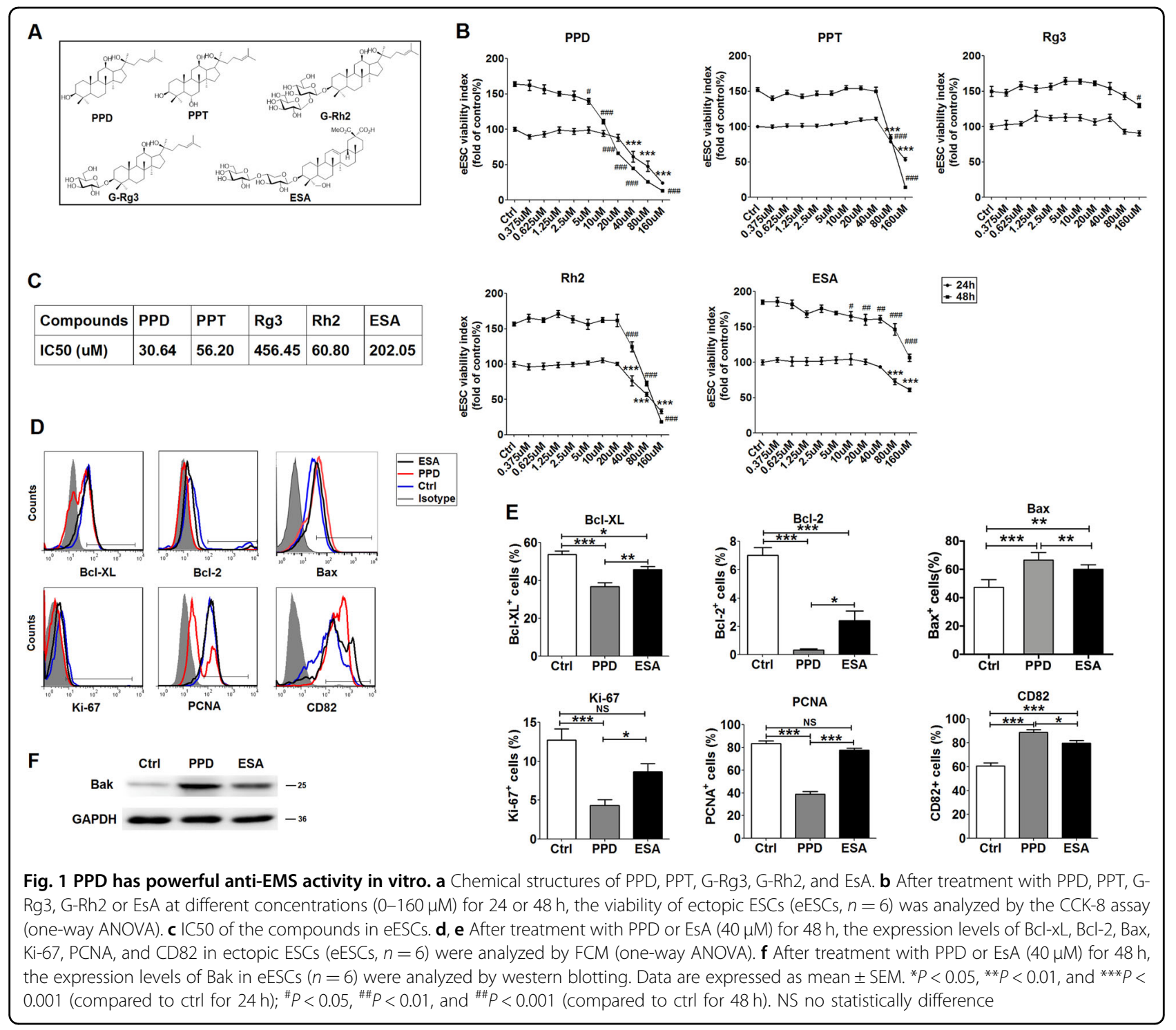

Meanwhile, treatment with PPD led to a significant decrease in ER $\alpha$ and increase in PR $\alpha$ in eESCs (Fig. 2c). PPD could restrict the stimulatory effect on ER $\alpha$ expression and inhibitory effect on autophagy induced by $17-\beta$ estradiol $\left(E_{2}\right)$ in eESCs (Fig. 2d, e). In addition, PPD alone, $\mathrm{ER} \alpha$ antagonist MPP dihydrochloride alone or the combination of PPD and MPP dihydrochloride resulted in the elevated autophagy of eESCs (Fig. 2f). In addition, MPP dihydrochloride led to a low level of Bcl-2/Bcl-XL and a high level of Bax/Bak, similar to that with PPD, but there was no synergistic effect with the combined treatment of MPP dihydrochloride plus PPD (Supplementary Figure 2). These data suggest that PPD can downregulate ER $\alpha$ expression and upregulate $\mathrm{PR} \alpha$ expression, and further induce a high level of autophagy and high ratio of proapoptosis molecules (Bax/Bak) to anti-apoptosis molecules (Bcl-2/Bcl-XL) in eESCs in vitro.

\section{PPD activates NK cells in response to eESCs in a co-culture model}

To explore the potential role of PPD in NK cells, NK cells were isolated from the peripheral blood of healthy women and were directly treated with PPD, or cocultured with control eESCs or PPD-pretreated eESCs. Next, we found that both PPD treatment and co-culture with PPD-pretreated eESCs increased the expression of natural killer group 2A (NKG2A) and activating natural cytotoxicity receptors (NKp30 and NKp46) ${ }^{26,27}$ and cytokine interferon (IFN) $-\gamma$, and decreased IL-10 expression in NK cells (Fig. 3a, b).

To further investigate the role of these NK cells in the viability of eESCs, we collected NK cells after co-culture with control eESCs (ESC-NK) or PPD-pretreated eESCs (ESC-PPD-NK), and then co-cultured with fresh eESCs (Fig. 3c). As shown, compared with ESC-NK co-cultured 


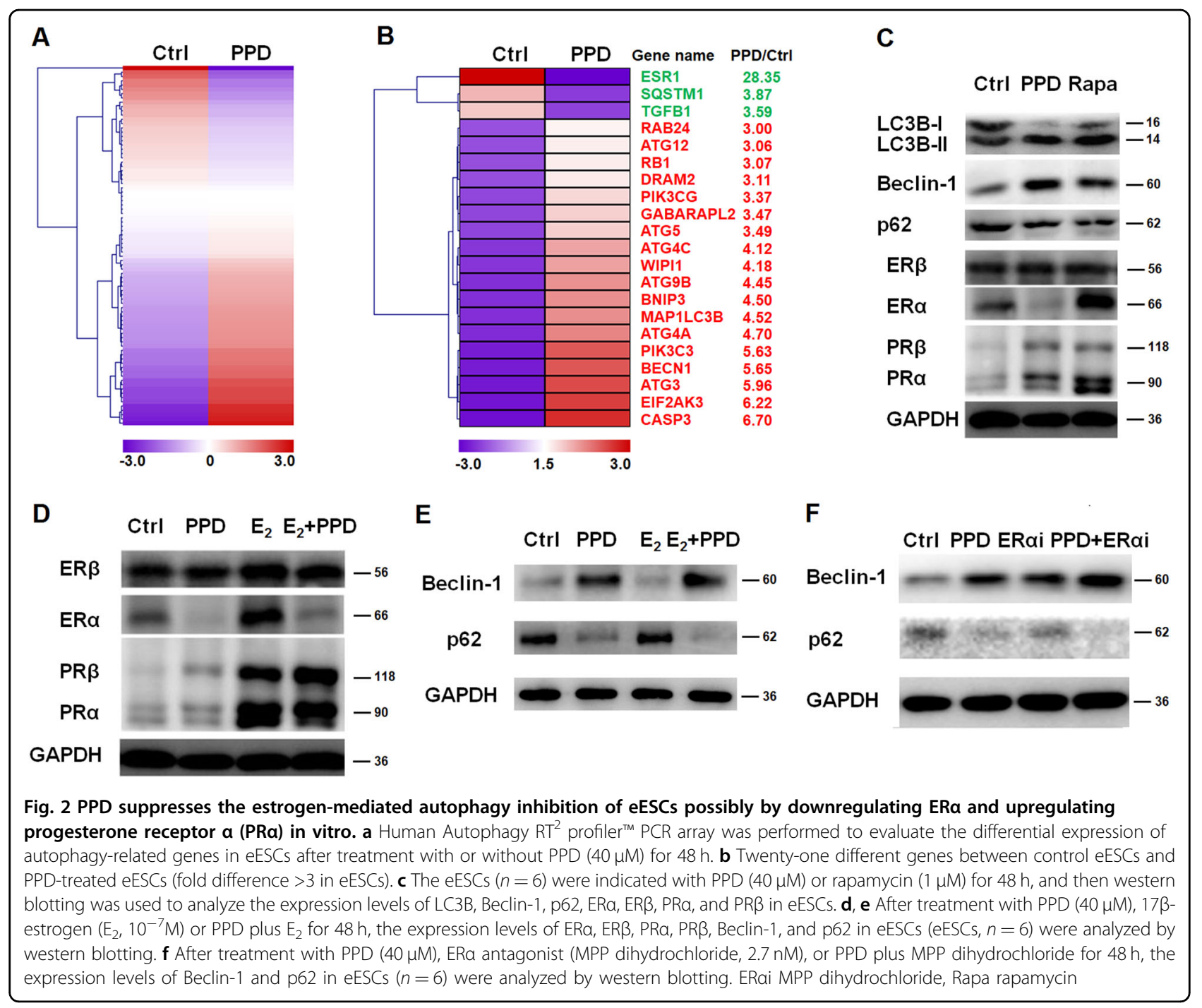

eESCs, the expression of Bcl-2, Bcl-xL, and $\mathrm{Ki}-67$ was markedly decreased, and the expression of CD82 was increased in ESC-PPD-NK co-cultured eESCs (Fig. 3d). These data suggest that PPD can induce the activation directly and indirectly by acting on eESCs, and PPDpretreated eESC-cultured NK cells may further restrict the growth and invasion in vitro.

Low doses of PPD do not influence ERa or PRa expression, proliferation, or the autophagy of the uterine endometrium in a mouse EMS model

To evaluate the potential therapeutic value of PPD in EMS, we analyzed the role of PPD in eutopic endometrium. According to the procedure of Figure 4a, the mouse EMS model was constructed by allotransplantation, and was intraperitoneally injected with low doses of PPD. As shown, PPD did not change the expression of $\mathrm{ER} \alpha, \mathrm{PR} \alpha$, Ki-67, Beclin-1, and LC3B in mouse uterine endometrium (Fig. 4b, c), suggesting that intraperitoneal injection of low-dose PPD does not regulate ER $\alpha$ and PR $\alpha$ expression, proliferation, and autophagy of uterine endometrium in a mouse EMS model.

PPD reverses the inhibitory effects of estrogen on the autophagy of mouse ectopic lesions and the activation of NK cells

As shown, all compounds (PPD, PPT, G-Rg3, G-Rh2, and EsA) at a high dose could significantly reduce the number and weight of ectopic lesions in a mouse EMS model, especially PPD (Fig. 5a). A significant inhibitory effect on the weight of mouse ectopic lesions could be achieved with the administration of low doses of PPD (Fig. 5b).

Subsequently, we found that PPD led to the decrease in $E R \alpha$ and the increase in PR $\alpha$ in ectopic lesions (Fig. 5c), echoing the results in vitro. $E_{2}$ significantly promoted the 


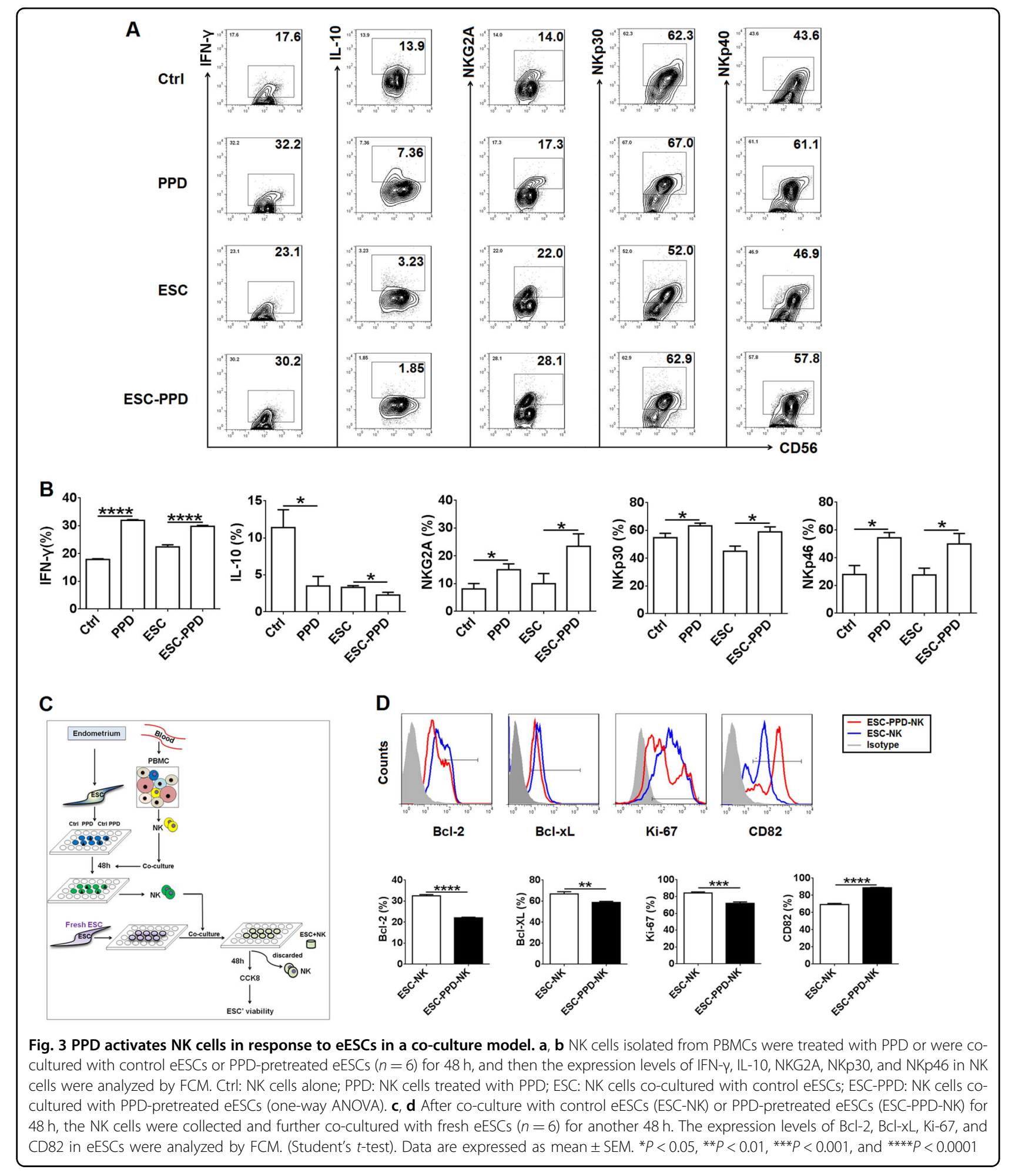

proliferation of ectopic lesions, and this effect could be reversed by PPD (Fig. 5 d). $E_{2}$ suppressed the expression of Beclin-1 and LC3B of ectopic lesions as well as 3methyladenine (3-MA, an autophagy inhibitor), and PPD also completely abrogated it (Fig. 5e, f).
In addition, treatment with $\mathrm{E}_{2}$ downregulated Granzyme $\mathrm{B}$ and NKG2D in $\mathrm{CD} 45^{+} \mathrm{CD} 3 \mathrm{e}^{-} \mathrm{DX} 5^{+} \mathrm{NK}$ cells from mouse peritoneal fluids (Fig. 6a, b). Although the intraperitoneal injection of PPD had no similarly effects on NK cells, the combination of PPD and $E_{2}$ 


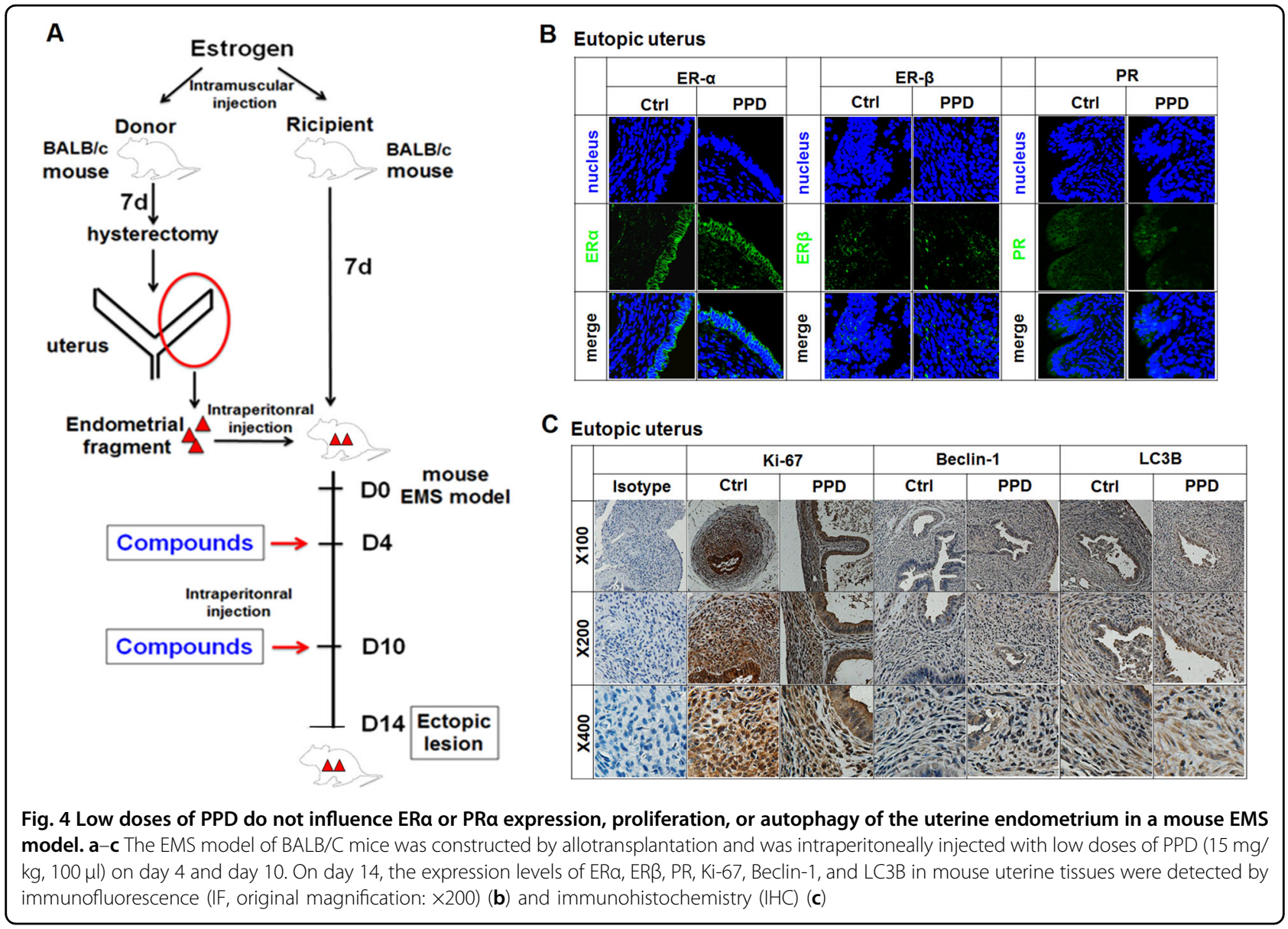

induced the activation of $\mathrm{NK}$ cells compared with $\mathrm{E}_{2}$ alone (Fig. 6a, b).

\section{PPD suppresses the estrogen-mediated growth of mouse ectopic lesions}

Finally, we analyzed the effect of PPD and $E_{2}$ on the number and weight of ectopic lesions in a mouse EMS model. As shown, $E_{2}$ increased the number and weight of mouse ectopic lesions (Fig. 7a, b). In contrast, PPD not only decreased the number and weight of mouse ectopic lesions, but also restricted the stimulatory effect of $E_{2}$ on the growth of mouse ectopic lesions (Fig. 7a, b). These data suggest that PPD suppresses the growth of mouse ectopic lesions induced by estrogen and plays a role in anti-mouse EMS activity.

\section{Discussion}

Ginsenosides within the dammarane-type consist mainly of three types classified according to their genuine aglycone moieties: PPD, PPT, and ocotillol. The potential health effects of ginsenosides include anti-inflammatory, antistress, anticarcinogenic, immunomodulatory, antiallergic, antiatherosclerotic, and antihypertensive effects as well as antidiabetic effects and regulatory effects on blood pressure and metabolism ${ }^{28-30}$. Here, we found that PPD, PPT, G-Rg3, and G-Rh2, especially PPD, could inhibit viability and growth as well as EsA both in vitro and in vivo. Additionally, low doses of PPD did not influence the growth of nESCs or the eutopic endometrium. These results suggest that PPD may be a safe and effective strategy for the treatment of EMS.

Autophagy has been linked to various pathophysiological processes, including cell death, development, tumorigenesis, and immunity ${ }^{31,32}$. We had found that autophagy in eESCs was significantly decreased, and estrogen suppressed the autophagy of ESCs by upregulating CXCL12 and CXCR4 expression. Here, the analysis of autophagy-related genes in this study showed that PPD enhanced the transcription of several autophagy-related genes in eESCs, such as ATGs and MAP1L3CB. Of note, the ESR1 and TGFB mRNA levels were decreased. Under hormonal control, TGF- $\beta$ is abundantly and differentially expressed in the endometrium and is involved in the promotion of ESC survival, cell adhesion and invasion, angiogenesis $^{33}$. Additionally, TGF- $\beta$ contributes to the suppression of the immune system in the environment of 


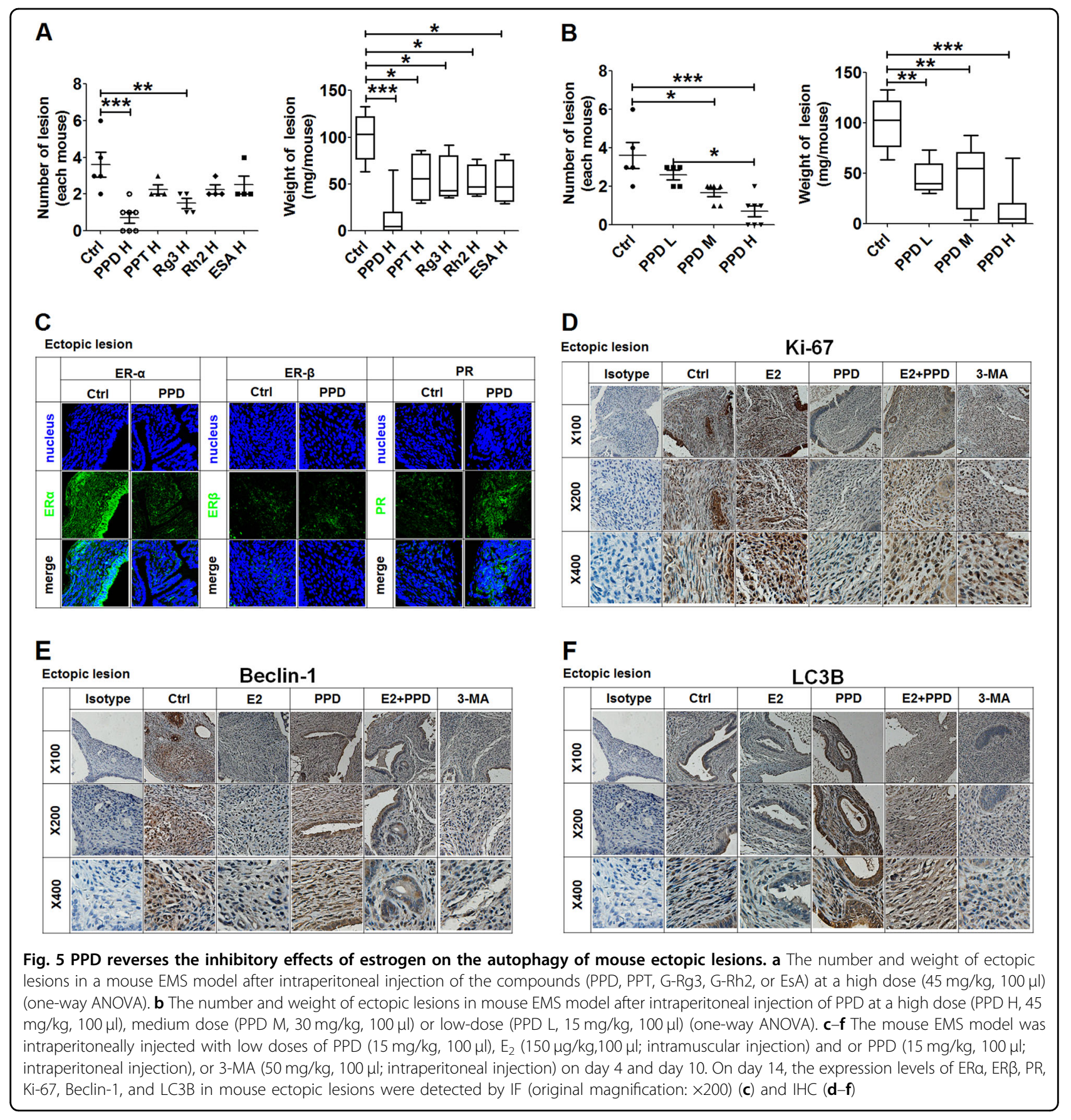

ectopic lesions such as the induction of IL- $10^{+} \mathrm{T}$ help (Th) 17 and regulatory $\mathrm{T}$ (Treg) cell differentiation and the impairment of NK cells cytotoxicity,33-35. Therefore, the anti-EMS effects of PPD may also be dependent on the regulation of TGFB.

Estrogen and progestogen modulate proliferation, apoptosis, and autophagy in the human endometrium and in endometriotic cells and tissues, further contributing to the origin and development of $\mathrm{EMS}^{7,36}$. In the current study, we also observed that E2 treatment led to increased
Ki-67 expression and a low autophagy level in ectopic lesions and elevated numbers and weights of ectopic lesions in a mouse EMS model. In addition, studies using ER knockout transgenic mice and treatment with ER subtype-selective ligands have indicated the crucial roles of ERs in EMS ${ }^{37-40}$. Progesterone resistance was observed in EMS. The molecular basis of progesterone resistance in EMS may be related to an overall reduction in the levels of $\mathrm{PR}^{37}$. The progesterone responses are mediated primarily via binding to and activation of the nuclear receptors (PR- 


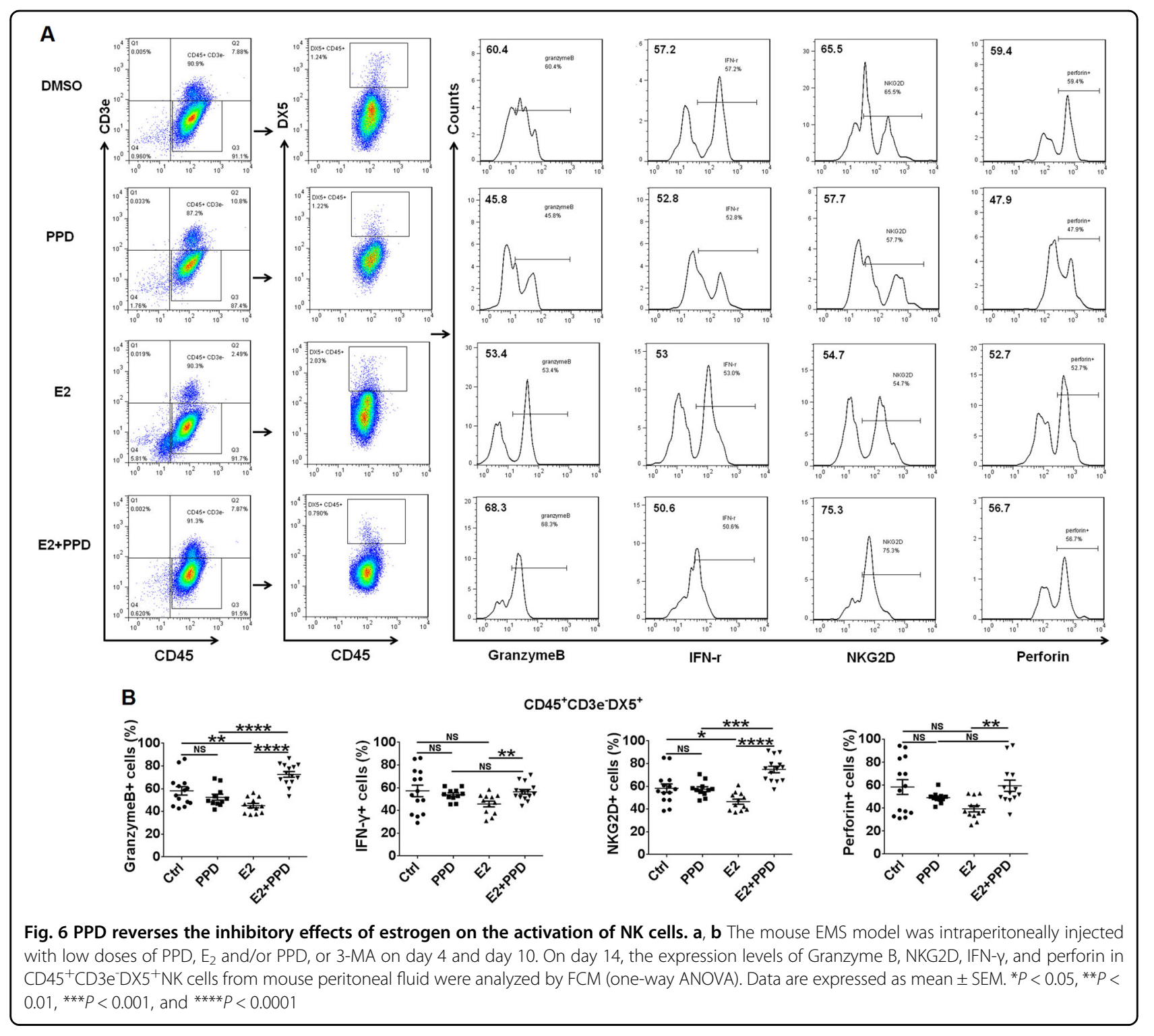

A and PR-B $)^{38,41}$. We found that PPD not only decreased $E R \alpha$ expression but also upregulated $P R \alpha$ expression in eESCs in vitro and in vivo. Our previous works have reported that progesterone signaling can suppress the inhibitory effect of estrogen on ESC autophagy ${ }^{7}$. Therefore, the stimulation of PR likely plays an important role in anti-EMS by PPD.

Here, we observed that PPD upregulated Bax and Bak, and downregulated Bcl-2 and Bcl-xL in ectopic ESCs possibly by downregulating ER $\alpha$. In addition to ER signaling, other signaling pathways such as PI3K/AKT, ROS and NF- $\mathrm{KB}$ participate in the regulation process of ginsenosides in cell apoptosis and death ${ }^{42-45}$. Under the regulation of estrogen, these signaling pathways are also involved in regulating ESC's apoptosis in $\mathrm{EMS}^{36,46-48}$. Therefore, PPD may also regulate these apoptosis-related proteins via these signaling pathways (e.g., PI3K/AKT, ROS, and NF- $\mathrm{kB}$ ) in EMS, a finding that require further research.

Ginsenosides are structurally described as triterpenoid saponins that contain a steroidal backbone. Although most naturally occurring ginsenosides have bulky sugar side chains that pose a huge steric hindrance for these molecules to bind to steroid receptors, various functional assays and molecular docking studies have provided evidence to show that ginsenosides can mediate their cellular activities by binding to the active sites of steroid receptors and downregulating the expression of estrogen receptors ${ }^{20,22,49}$. On the other hand, other studies suggest that ginsenosides may modulate their cellular actions via pathways independent of steroid receptors ${ }^{50-52}$. However, the regulatory mechanism of ginsenosides for 

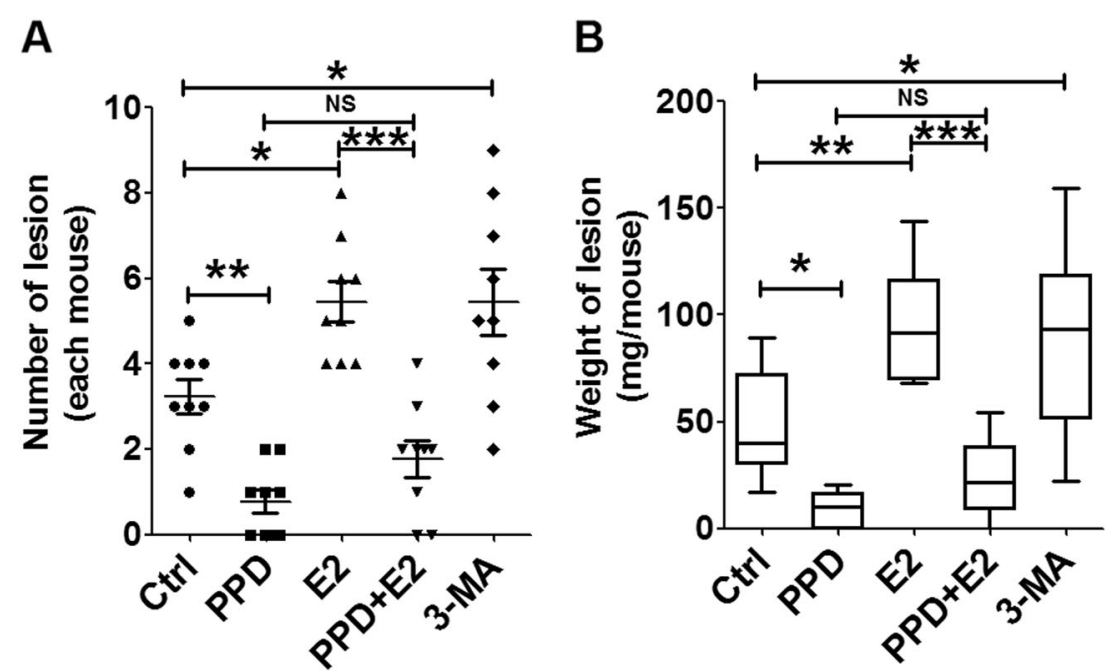

Fig. 7 PPD suppresses the estrogen-mediated growth of mouse ectopic lesions. $\mathbf{a}$, $\mathbf{b}$ Number and weight of ectopic lesions from the mouse EMS model, which was treated as described in Figure 6. Data are expressed as mean \pm SEM. ${ }^{*} P<0.05,{ }^{* *} P<0.01$, and ${ }^{* * *} P<0.001$

hormone receptors remain unclear and warrants further research.

The impaired cytotoxic activity of NK cells is associated with several physiological and pathological processes, including EMS ${ }^{14,27,53}$. The effector functions of NK cells include the cytotoxicity and secretion of cytokines (e.g., IFN- $\gamma$, TNF- $\alpha$ ). The most potent activated receptors of NK cells are the antibody-dependent cell-mediated cytotoxicity (ADCC)-mediating molecule CD16 and NKG2D ${ }^{26,27}$. Moreover, NK cells recognize their ligands in tumor or virus-infected cells and mediate natural cytotoxicity through a set of activating natural cytotoxicity receptors (e.g., NKp30, and NKp46) ${ }^{26,27}$. In the current study, PPD promoted the cytotoxic activity of NK cells, such as via the upregulation of granzyme B and perforin, especially under the stimulation of estrogen. Of note, we did not observe the effect of PPD alone on the activation NK cells in the mouse EMS model. This result may have resulted from the low concentration and or complex microenvironment in EMS, but the mechanisms deserve further investigation.

EMS is also an inflammatory disorder. The action of estrogen not only is essential for endometriotic tissue growth but also contributes to ongoing inflammation, neovascularization, and associated pain ${ }^{54}$. Ginsenosides are known to have anti-cancer properties based on their anti-inflammatory activation, and their low toxicities render them excellent candidates for cancer therapy ${ }^{20}$. For example, ginseng total saponins markedly reduce the production of pro-inflammatory cytokines (e.g., TNF- $\alpha$, interleukin (IL)-1 $\beta$, and IL-6) in lipopolysaccharide (LPS)stimulated rat astrocyte and microglia cultures in vitro. These inflammatory cytokines were also increased in the
eESCs, ectopic lesions and peritoneal fluids from patients with $\mathrm{EMS}^{40,55}$. Therefore, the anti-EMS property may be partly dependent on the anti-inflammatory effects, which requires further research.

Collectively, our findings, schematized in Figure 8, reveal that PPD can downregulate the expression of ER $\alpha$ and upregulate the expression of PR $\alpha$. The inhibition of estrogen/ER $\alpha$ signaling mediated by PPD further induces the autophagy of eESCs, activates the cytotoxicity of NK cells in response to eESCs, suppresses the growth of ectopic lesions, enhances the immune surveillance of ectopic lesions, and finally inhibits the development of EMS. Therefore, PPD should have potential therapeutic value for treating diseases such as EMS. Differences in the saponin structure markedly influence bioactivity and bioavailability. Therefore, further structural modification of PPD is needed to identify better anti-EMS drugs.

\section{Materials and methods}

\section{Patients and sample collection}

The study protocol was approved by the Human Research Ethics Committee of Obstetrics and Gynecology Hospital, Fudan University, and written informed consent was obtained from all patients. All of the endometriotic tissues were obtained by laparoscopy from 43 patients with EMS at the Obstetrics and Gynecology Hospital of Fudan University. Normal endometrium was obtained through hysterectomy from patients with leiomyoma (six cases) as healthy control samples. None of the included patient experienced complications related to pelvic inflammatory disease and no patient took any medication or received hormonal therapy within six months prior to surgery. All the samples were obtained in the proliferation 


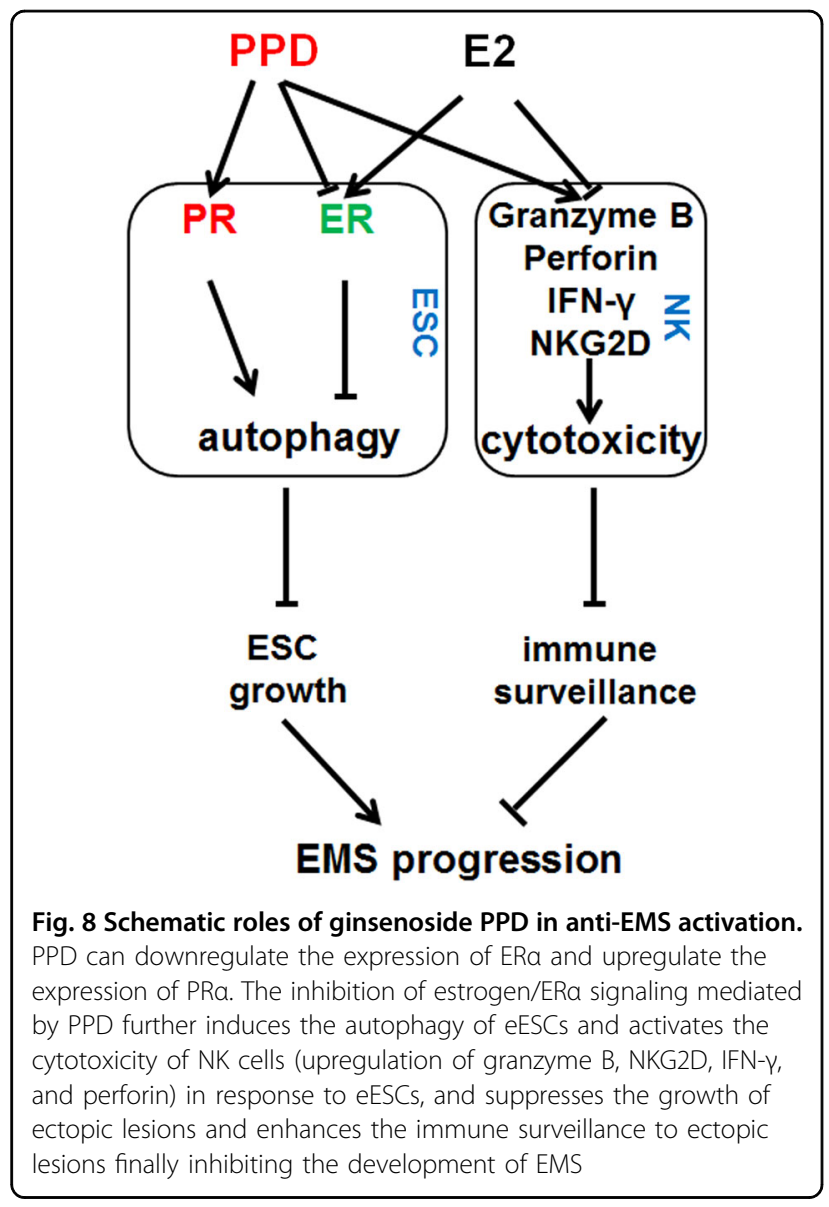

phase of the cell cycle, as confirmed histologically according to established criteria.

\section{Isolation and culture of ESCs}

We isolated human $\mathrm{nESCs}$ from the endometrium of healthy control subjects, and eESCs from ectopic lesions of women with EMS according to a previously described method $^{25}$. It supplied $>98 \%$ vimentin ${ }^{+} \mathrm{CK} 7^{-}$ESCs, as confirmed by flow cytometry (FCM) analysis.

\section{Purification of NK cells}

Peripheral blood mononuclear cells (PBMCs) were isolated from healthy fertile women. Human NK cells were isolated from PBMCs using magnetic beads (Miltenyi Biotec, Bergisch Gladbach, Germany) for in vitro experiments. These NK cells were directly treated with PPD ( $40 \mu \mathrm{M}$, Sigma-Aldrich Co. LLC., USA); or were cocultured with control eESCs or PPD-pretreated eESCs for $48 \mathrm{~h}$, and then these NK cells were collected to analyze the expression of IFN- $\gamma$, IL-10, NKG2A, NKp30, and $\mathrm{NKp} 40$ by FCM; or were further co-cultured with fresh eESCs for another $48 \mathrm{~h}$, and then the expression levels of Bcl-2, Bcl-xL, Ki-67, and CD82 in eESCs were analyzed by FCM.

\section{The cell-counting kit-8 (CCK-8) assay}

The eESCs were treated with PPD, PPT, G-Rg3, G-Rh2, or EsA (0-160 $\mu \mathrm{M}$, Sigma-Aldrich Co. LLC., USA) for 24 or $48 \mathrm{~h}$, with $0.1 \%$ DMSO as a blank control. Next, these cells were collected, and the viability was detected by the CCK-8 assay (Dojindo, Japan). According to the manufacturer's protocol, the CCK-8 reagent was added to each well and cells were incubated at $37^{\circ} \mathrm{C}$ for $1-4 \mathrm{~h}$. The absorbance (optical density) at $450 \mathrm{~nm}$ was measured and used to represent the cell viability. Each experiment was performed in six parallel wells and repeated three times.

\section{FCM}

After treatment with PPD $(40 \mu \mathrm{M})$ or EsA $(40 \mu \mathrm{M})$, or co-culture with control eESCs (ESC-NK) or PPDpretreated eESCs (ESC-PPD-NK), these eESCs were collected and the expression levels of Bcl-2, Bcl-xL, Bax, Ki67, PCNA, and or CD82 (all from Biolegend, USA) were analyzed by FCM according to the manufacturer's instructions. In addition, the expression levels of IFN- $\gamma$, IL-10, NKG2A, NKp30, and NKp40 (all from Biolgend) in CD $56^{+} \mathrm{NK}$ cells were analyzed by FCM. Isotypic IgG antibodies were used as controls. The samples were analyzed using a FACS-Calibur flow cytometer (Becton Dickinson, USA) and Cellquest software (Becton Dickinson). Statistical analysis was conducted using isotypematched controls as references.

\section{Human Autophagy $\mathbf{R T}^{2}$ profiler ${ }^{\mathrm{TM}} \mathrm{PCR}$ array}

The eESCs were indicated with PPD $(40 \mu \mathrm{M})$ for $48 \mathrm{~h}$, with $0.1 \%$ DMSO as a blank control. Next, human Autophagy $\mathrm{RT}^{2}$ profiler ${ }^{\text {rm }}$ PCR array (Catalog No. PAHS084ZR, 96-well format) was utilized to analyze the transcriptional levels of these autophagy-related genes in eESCs as described previously ${ }^{7,56}$. The plates were processed using an Applied Biosystems 7500 fast RT-PCR system (Applied Biosystems, USA), and the data were interpreted with SABiosciences' web-based PCR array analysis tool.

\section{Protein extraction and western blotting}

The eESCs were treated with PPD $(40 \mu \mathrm{M})$, rapamycin ( $1 \mu \mathrm{M}$, sigma), E2 (10- ${ }^{7} \mathrm{M}$, Sigma), E2 plus PPD, ER $\alpha$ antagonist (MPP dihydrochloride; $2.7 \mathrm{nM}$; Tocris Bioscience, USA), or PPD plus MPP dihydrochloride for $48 \mathrm{~h}$, and then cells were washed in phosphate buffered saline (PBS), detached with a cell scraper and centrifuged for $20 \mathrm{~min}$ at $12,000 \mathrm{r} . p . \mathrm{m}$. at $4{ }^{\circ} \mathrm{C}$. The pellet was resuspended in high efficiency cell tissue rapid lysis buffer (RIPA; Beyotime, Shanghai, China) containing 1\% phenylmethanesulfonylfluoride (PMSF; Beyotime) proteinase and $1 \%$ phosphatase inhibitors (Roche, USA). Cell lysates were boiled for $10 \mathrm{~min}$ at $95^{\circ} \mathrm{C}$ and then were stored at $-80^{\circ} \mathrm{C}$. Protein concentrations were quantified using the 
BCA protein assay kit (Beyotime). Total proteins $(20 \mu \mathrm{g})$ were electrophoresed in SDS-PAGE gels (EpiZyme scientific) using a Miniprotein III system (Bio-Rad, USA) and were transferred to PVDF membranes (Millipore, USA) at $2 \mathrm{~h}$, followed by overnight incubation with primary antibody against Bax, Bak, Beclin-1, p62, LC3B, ER $\alpha$, PR, or GAPDH (1:1000; Cell Signaling Technology, USA), and ER $\beta$ (1:200; Santa Cruz Biotechnology, Santa Cruz, CA, USA) at $4{ }^{\circ} \mathrm{C}$. Then PVDF membranes were washed three times with PBST solution and were incubated at room temperature for $1 \mathrm{~h}$ in peroxidase-conjugated goat antirabbit IgG secondary antibodies (1:5000; Bioworld Technology, Co. Ltd. USA). Thereafter the membrane was washed three times and processed for chemiluminescence using the Immobilon Western Chemiluminescent HRP Substrate Kit (Millipore).

\section{Mouse EMS model}

A group of adult female BALB/C mice was purchased from the Laboratory Animal Facility of Fudan University and was used for this study. They were maintained for 2 weeks at the animal facility before use. The Animal Care and Use Committee of Shanghai First Maternity and Infant Hospital, Tongji University School of Medicine approved all the animal protocols.

We constructed an intraperitoneal EMS model. On Day 0 , the uterus of female BALB/C mice (Donor mice) was minced, and then the tissue debris was intraperitoneally injected into female BALB/C mice (for recipient mice, the ratio of the uterus to intraperitoneal injection of mice was 1:2). On day 4 and day 10, the EMS mice were intraperitoneally injected with the compounds (PPD, PPT, G-Rg3, G-Rh2, or EsA) at a high dose $(45 \mathrm{mg} / \mathrm{kg}, 100 \mu \mathrm{l})$, medium dose $(30 \mathrm{mg} / \mathrm{kg}, 100 \mu \mathrm{l})$ or low-dose $(15 \mathrm{mg} / \mathrm{kg}, 100 \mu \mathrm{l})$. In addition, some EMS mice were treated with $\mathrm{E}_{2}(150 \mu \mathrm{g} /$ $\mathrm{kg}, 100 \mu \mathrm{l}$; intramuscular injection), and/or PPD (15 mg/ $\mathrm{kg}, 100 \mu \mathrm{l}$; intraperitoneally injection), or $3-\mathrm{MA}(50 \mathrm{mg} /$ $\mathrm{kg}, 100 \mu \mathrm{l}$; intraperitoneally injection), 0.1\% DMSO (100 $\mu$ l) as a control. On Day 14, the EMS-like lesions and peritoneal fluids were collected and detected. The number and weight of EMS-like lesions were counted. Immunohistochemistry (IHC) and immunofluorescence (IF) were used to analyze the expression of ER $\alpha$ ER $\beta, P R, K i-67$, Beclin-1 and LC3B in the EMS-like lesions. Additionally, the expression levels of IFN- $\gamma$, NKG2D, granzyme B and perforin (all from Biolegend) in $\mathrm{CD} 45^{+} \mathrm{CD} 3 \mathrm{e}^{-} \mathrm{DX} 5^{+} \mathrm{NK}$ cells in peritoneal fluid were analyzed by FCM.

\section{IHC and IF}

For IHC, paraffin sections $(5 \mu \mathrm{m})$ of the EMS-like lesions and uterine endometrium were dehydrated in graded ethanol and then were incubated with hydrogen peroxide and $1 \%$ bovine serum albumin/TBS to block endogenous peroxidase. The samples were then incubated with rabbit anti-human Ki-67 (1:500; Abcam, USA), Beclin-1 (1:200; Abcam) and LC3B (1:500; Abcam) or rabbit IgG isotype (Abcam) overnight at $4{ }^{\circ} \mathrm{C}$ in a humid chamber. After washing three times with TBS, the sections were overlaid with peroxidase-conjugated goat antirabbit IgG, and the reaction was developed with 3,3-diaminobenzidine (DAB) and counterstained with hematoxylin.

For IF, according to a previous procedure ${ }^{7}$, EMS-like lesions and uterine endometrium tissues were incubated with anti-goat $\mathrm{ER} \alpha$, anti-goat $\mathrm{ER} \beta$, anti-goat PR antibody (1:100, Abcam) in PBS at $4{ }^{\circ} \mathrm{C}$ overnight. The slides were then incubated with Alexa Fluor 488-conjugated donkey anti-goat secondary antibody (1:500; Abcam). The nuclei were then stained with 4',6-diamidino-2-phenylindole (DAPI; Beyotime, China). Images were captured with a confocal microscope (Leica, Germany).

\section{Statistics}

All values are shown as the mean \pm SEM. The data were analyzed with GraphPad Prism version 5 by $t$-test for two groups or one-way analysis of variance using Tukey's post hoc test for multiple groups. Differences were considered statistically significant at $P<0.05$.

\section{Acknowledgements \\ We thank Dr. Yi-Qin Wang in the Department of Pathology, Hospital of Obstetrics and Gynecology, Fudan University for helping with histological analysis. This study was supported by the Major Research Program of National Natural Science Foundation of China (NSFC) (nos. 91542108, 81471513, 31671200, 81601354, and 81671460), the Shanghai Rising-Star Program 16QA1400800, the Development Fund of Shanghai Talents (201557), the Oriented Project of Science and Technology Innovation from Key Lab. of Reproduction Regulation of NPFPC (CX2017-2), the National Basic Research Program of China (2015CB943300), the National Key Research and Development Program of China (2017YFC1001401), the Program for Shanghai leaders, the Program of Shanghai Outstanding Academic Leader (15XD1500900), the National Science Foundation of Jiangsu Province (no. BK20160128), and Fundamental Research Funds for the Central Universities (no. 021414380180).}

\begin{abstract}
Author details
'Laboratory for Reproductive Immunology, Key Laboratory of Reproduction Regulation of NPFPC, SIPPR, IRD, Hospital of Obstetrics and Gynecology, Fudan University Shanghai Medical College, 200011 Shanghai, China. ${ }^{2}$ National Research Centre for Carbohydrate Synthesis, Jiangxi Normal University, 330022 Jiangxi, Nanchang, China. ${ }^{3}$ Wallace H.Coulter Department of Biomedical Engineering, Georgia Tech College of Engineering and Emory School of Medicine, Georgia Institute of Technology, Atlanta 30332 GA, USA. ${ }^{4}$ Clinical and Translational Research Center, Shanghai First Maternity and Infant Hospital, Tongji University School of Medicine, 200040 Shanghai, China. ${ }^{5}$ Shanghai Key Laboratory of Female Reproductive Endocrine Related Diseases, 200011 Shanghai, China
\end{abstract}

Authors' contributions

B.Z. and W.J.Z. conducted all experiments and prepared the figures and the manuscript. C.J.G., K.W., H.L.Y., and J.J.Y. assisted with animal trials and IHC assay. J.M. and X.F.H. helped to edit the manuscript. J.S.S. helped to design the study and edit the manuscript. F.Y.X. and D.J.L. helped to edit the manuscript. M.Q.L. and L.P.J. designed the study, supervised the project, and edited the manuscript. All the authors were involved in writing the manuscript. 


\section{Conflict of interest}

The authors declare that they have no conflict of interest.

\section{Publisher's note}

Springer Nature remains neutral with regard to jurisdictional claims in published maps and institutional affiliations.

Supplementary Information accompanies this paper at (https://doi.org/ 10.1038/s41419-018-0581-2).

Received: 27 November 2017 Revised: 5 April 2018 Accepted: 10 April 2018 Published online: 14 May 2018

\section{References}

1. Bulun, S. E. Endometriosis. N. Engl. J. Med. 360, 268-279 (2009).

2. Giudice, L. C. \& Kao, L. C. Endometriosis. Lancet 364, 1789-1799 (2004).

3. Han, S. J. et al. A new isoform of steroid receptor coactivator-1 is crucial for pathogenic progression of endometriosis. Nat. Med 18, 1102-1111 (2012).

4. Eskenazi, B. \& Warner, M. L. Epidemiology of endometriosis. Obstet. Gynecol. Clin. North Am. 24, 235-258 (1997).

5. Pritts, E. A. \& Taylor, R. N. An evidence-based evaluation of endometriosisassociated infertility. Endocrinol. Metab. Clin. North Am. 32, 653-667 (2003).

6. Pollacco, J., Sacco, K, Portelli, M., Schembri-Wismayer, P. \& Calleja-Agius, J. Molecular links between endometriosis and cancer. Gynecol. Endocrinol. 28, 577-581 (2012)

7. Mei, J. et al. Estrogen promotes the survival of human secretory phase endometrial stromal cells via CXCL12/CXCR4 up-regulation-mediated autophagy inhibition. Hum. Reprod. 30, 1677-1689 (2015).

8. $\mathrm{Yu}$, J. J. et al. IL15 promotes growth and invasion of endometrial stromal cells and inhibits killing activity of NK cells in endometriosis. Reproduction 152, 151-160 (2016).

9. Yang, H. L. et al. The crosstalk between endometrial stromal cells and macrophages impairs cytotoxicity of NK cells in endometriosis by secreting IL-10 and TGF- $\beta$. Reproduction 154, 815-825 (2017).

10. He, C. \& Klionsky, D. J. Regulation mechanisms and signaling pathways of autophagy. Annu. Rev. Genet. 43, 67-93 (2009).

11. Choi, J., Jo, M., Lee, E., Oh, Y. K. \& Choi, D. The role of autophagy in human endometrium. Biol. Reprod. 86, 70 (2012).

12. Choi, J., Jo, M., Lee, E., Kim, H. J. \& Choi, D. Differential induction of autophagy by $\mathrm{MTOR}$ is associated with abnormal apoptosis in ovarian endometriotic cysts. Mol. Hum. Reprod. 20, 309-317 (2014).

13. Choi, S., Shin, H., Song, H. \& Lim, H. J. Suppression of autophagic activation in the mouse uterus by estrogen and progesterone. J. Endocrinol. 221, 39-50 (2014).

14. Yang, H. L. et al. Autophagy in endometriosis. Am. J. Transl. Res. 9, 4707-4725 (2017).

15. Ruiz, A. et al. Effect of hydroxychloroquine and characterization of autophagy in a mouse model of endometriosis. Cell Death Dis. 7, e2059 (2016).

16. Wu, X. et al. Human first-trimester trophoblast cells recruit CD56brightCD16NK cells into decidua by way of expressing and secreting of CXCL12/stromal cell-derived factor 1. J. Immunol. 175, 61-68 (2005).

17. Meng, W., Xue, S. \& Chen, Y. The role of CXCL12 in tumor microenvironment. Gene 641, 105-110 (2018).

18. Christensen, L. P. Ginsenosides chemistry, biosynthesis, analysis, and potential health effects. Adv. Food Nutr. Res. 55, 1-99 (2009).

19. Zhang, Q., Kang, X. \& Zhao, W. Antiangiogenic effect of low-dose cyclophosphamide combined with ginsenoside Rg3 on Lewis lung carcinoma. Biochem. Biophys. Res. Commun. 342, 824-828 (2006).

20. Wong, A. S., Che, C. M. \& Leung, K. W. Recent advances in ginseng as cancer therapeutics: a functional and mechanistic overview. Nat. Prod. Rep. 32, 256-272 (2015).

21. Li, B. et al. Ginsenoside Rh2 induces apoptosis and paraptosis-like cell death in colorectal cancer cells through activation of p53. Cancer Lett. 301, 185-192 (2011).

22. Gu, C. J. et al. Protopanaxadiol and metformin synergistically inhibit estrogenmediated proliferation and anti-autophagy effects in endometrial cancer cells. Am. J. Transl. Res. 9, 4071-4082 (2017).
23. $\mathrm{Xu}, \mathrm{F}$. Y. et al. The antitumor activity study of ginsenosides and metabolites in lung cancer cell. Am. J. Transl. Res. 8, 1708-1718 (2016).

24. Wu, F. Yi, Y., Sun, P. \& Zhang, D. Synthesis, in vitro inhibitory activity towards COX-2 and haemolytic activity of derivatives of esculentoside A. Bioorg. Med. Chem. Lett. 17, 6430-6433 (2017).

25. Li, M. Q. et al. CD82 gene suppression in endometrial stromal cells leads to increase of the cell invasiveness in the endometriotic milieu. J. Mol. Endocrinol. 47, 195-208 (2011).

26. Cooper, M. A. et al. Human natural killer cells: a unique innate immunoregulatory role for the CD56 (bright) subset. Blood 97, 3146-3151 (2001).

27. Lanier, L. L. NK cell recognition. Annu. Rev. Immunol. 23, 225-227 (2005).

28. Chang, Y. S., Seo, E. K. Gyllenhaal, C. \& Block, K. I. Panax ginseng: a role in cancer therapy? Integr. Cancer Ther. 2, 13-33 (2003).

29. Attele, A. S., Wu, J. A. \& Yuan, C. S. Ginseng pharmacology: multiple constituents and multiple actions. Biochem. Pharmacol. 58, 1685-1693 (1999).

30. Kitts, D. D. \& Hu, C. Efficacy and safety of ginseng. Public Health Nutr. 3, 473-485 (2000).

31. Levine, B., Mizushima, N. \& Virgin, H. W. Autophagy in immunity and inflammation. Nature 469, 323-335 (2011).

32. Auberger, P. \& Puissant, A. Autophagy, a key mechanism of oncogenesis and resistance in leukemia. Blood 129, 547-552 (2017).

33. Omwandho, C. O., Konrad, L., Halis, G., Oehmke, F. \& Tinneberg, H. R. Role of TGF-betas in normal human endometrium and endometriosis. Hum. Reprod. 25, 101-109 (2010).

34. Chang, K. K. et al. IL-27 triggers IL-10 production in Th17 cells via a C-Maf/ RORYt/Blimp-1 signal to promote the progression of endometriosis. Cell Death Dis. 8, e2666 (2017).

35. Li, M. Q. et al. CD4+Foxp3+regulatory $T$ cell differentiation mediated by endometrial stromal cell-derived TECK promotes the growth and invasion of endometriotic lesions. Cell Death Dis. 5, e1436 (2014).

36. Reis, F. M., Petraglia, F. \& Taylor, R. N. Endometriosis: hormone regulation and clinical consequences of chemotaxis and apoptosis. Hum. Reprod. 19, 406-418 (2013).

37. Bulun, S. E. et al. Estrogen receptor-beta, estrogen receptor-alpha, and progesterone resistance in endometriosis. Semin Reprod. Med. 28, 36-43 (2010).

38. Kulak, J. Jr., Fischer, C., Komm, B. \& Taylor, H. S. Treatment with bazedoxifene, a selective estrogen receptor modulator, causes regression of endometriosis in a mouse model. Endocrinology 152, 3226-3232 (2011).

39. Harris, H. A., Bruner-Tran, K. L., Zhang, X., Osteen, K. G. \& Lyttle, C. R. A selective estrogen receptor-beta agonist causes lesion regression in an experimentally induced model of endometriosis. Hum. Reprod. 20, 936-941 (2005).

40. Zhao, Y. et al. Dual suppression of estrogenic and inflammatory activities for targeting of endometriosis. Sci. Transl. Med. 7, 271 ra9 (2015).

41. Al-Sabbagh, M., Lam, E. W. \& Brosens, J. J. Mechanisms of endometrial progesterone resistance. Mol. Cell. Endocrinol. 358, 208-215 (2012).

42. $\mathrm{Wu}, \mathrm{Q}$. et al. Ginsenoside Rh4 induces apoptosis and autophagic cell death through activation of the ROS/JNK/p53 pathway in colorectal cancer cells. Biochem. Pharmacol. 148, 64-74 (2018).

43. Cui, J. et al. Ginsenoside Rg2 protects PC12 cells against $\beta$-amyloid25-35induced apoptosis via the phosphoinositide 3-kinase/Akt pathway. Chem. Biol. Interact. 275, 152-161 (2017).

44. Yuan, Z., Jiang, H., Zhu, X., Liu, X. \& Li, J. Ginsenoside Rg3 promotes cytotoxicity of Paclitaxel through inhibiting NF-KB signaling and regulating $\mathrm{Bax} / \mathrm{BCl}-2$ expression on triple-negative breast cancer. Biomed. Pharmacother. 89, 227-232 (2017).

45. Huang, G. et al. Notoginsenoside R1 attenuates glucose-induced podocyte injury via the inhibition of apoptosis and the activation of autophagy through the PI3K/Akt/mTOR signaling pathway. Int. J. Mol. Med 39, 559-568 (2017).

46. Li, M. Q. et al. CXCL8 enhances proliferation and growth and reduces apoptosis in endometrial stromal cells in an autocrine manner via a CXCR1triggered PTEN/AKT signal pathway. Hum. Reprod. 27, 2107-2116 (2012).

47. Park, S., Lim, W., Bazer, F. W. \& Song, G. Apigenin induces ROS-dependent apoptosis and ER stress in human endometriosis cells. J. Cell. Physiol. 233, 3055-3065 (2018).

48. Banu, S. K., Lee, J., Speights, V. O. Jr., Starzinski-Powitz, A. \& Arosh, J. A. Selective inhibition of prostaglandin E2 receptors EP2 and EP4 induces apoptosis of human endometriotic cells through suppression of ERK1/2, AKT, NFkappaB, and beta-catenin pathways and activation of intrinsic apoptotic mechanisms. Mol. Endocrinol. 23, 1291-1305 (2009).

49. Park, J. et al. Effects of ginseng on two main sex steroid hormone receptors: estrogen and androgen receptors. J. Ginseng Res. 41, 215-221 (2017). 
50. Leung, K. W. et al. Signaling pathway of ginsenoside-Rg1 leading to nitric oxide production in endothelial cells. FEBS Lett. 580, 3211-3216 (2006).

51. Song, I. H. \& Buttgereit, F. Non-genomic glucocorticoid effects to provide the basis for new drug developments. Mol. Cell. Endocrinol. 246, 142-146 (2006).

52. Hafezi-Moghadam, A. et al. Acute cardiovascular protective effects of corticosteroids are mediated by non-transcriptional activation of endothelial nitric oxide synthase. Nat. Med. 8, 473-479 (2002).
53. Osuga, Y. et al. Lymphocytes in endometriosis. Am. J. Reprod. Immunol. 65 1-10 (2011).

54. Bulun, S. E. et al. Role of estrogen receptor- $\beta$ in endometriosis. Semin. Reprod. Med. 30, 39-45 (2012).

55. Burney, R. O. \& Giudice, L. C. Pathogenesis and pathophysiology of endometriosis. Fertil. Steril. 98, 511-519 (2012).

56. Deshpande, N. P. et al. Campylobacter concisus pathotypes induce distinct global responses in intestinal epithelial cells. Sci. Rep. 6, 34288 (2016). 